ジョイント・シンポジウム 3

\title{
Implication of xanthine oxidase-inhibiting therapy for non-gouty conditions
}

Masayuki Hakoda

Association of serum urate levels with the development and mortality of cardiovascular diseases has been implicated for many years. This association has been usually more potent for women than for men. Such phenomenon might reflect non-direct effects of urate on cardiovascular pathophysiology, at least in humans. This notion is supported by several findings including the one that direct infusion of urate into human vessels showed no effects on vascular endothelial function and blood pressure. Recently, results of several observational and randomized studies that include interventional process have been appearing. In most cases, allopurinol has been used for such interventions.

Allopurinol has been suggested effective in the management of patients with heart failure in several experimental and observational studies. These beneficial effects may be due to its inhibitory effects on the production of oxygen free radicals from xanthine oxidase, but not due to its urate-lowering effects, because vascular endothelial function of heart failure patients improved by taking allopurinol but not by a uricosuric drug, probenecid. A recent randomized double blind study, however, did not show any significant effects of oxypurinol on the prognosis of heart failure. The dose of oxypurinol used in this study was $600 \mathrm{mg}$, corresponding to an allopurinol dose of $81 \mathrm{mg}$, which might have been insufficient. This notion is based on the results of retrospective observational studies showing that $\geq 300 \mathrm{mg}$ allopurinol is more effective than lower doses. Another observational study suggested that treatment with allopurinol decreased hospitalization or death in heart failure patients with gout. A recent double-blind crossover study suggested that allopurinol may be effective in patients with angina pectoris. They showed that $600 \mathrm{mg}$ allopurinol prolonged time to the appearance of ST-depression or chest pain during exercise as compared to placebo among patients with stable angina. These results implicate beneficial effects of xanthine oxidase-inhibition on the management of cardiovascular diseases.

Department of nutritional sciences, Faculty of Human Ecology, Yasuda Women's University 